\title{
Impacto de las donaciones y transferencias en la inversión pública en la región Puno, periodo 2009-2019
}

\author{
Julio Cesar Quispe Mamani \\ jcquispe@unap.edu.pe \\ Universidad Nacional del Altiplano \\ Octavio Benigno Torres Pari \\ Serrot_1@ hotmail.es \\ Universidad Andina Néstor Cáceres Velásquez \\ Ilsse Bedoya Gómez \\ ilssebedoya@gmail.com \\ Universidad Nacional del Altiplano \\ Maria Isabel Alegre Larico \\ mialsud@gmail.com \\ Universidad Andina Néstor Cáceres Velásquez \\ Elisalde Coacalla Vargas \\ ecoacalla@unap.edu.pe \\ Puno - Perú
}

\section{RESUMEN}

El objetivo de la investigación fue determinar el impacto de las donaciones y transferencias en la inversión pública de la región Puno 2009-2019, en vista que en los últimos años, la inversión pública ha sido cuestionada principalmente porque ésta no ha mostrado mejoras significativas en el desarrollo de la región de Puno. Se aplicó una metodología de tipo correlacional, descriptiva, considerando la base de datos de series temporales para el análisis del impacto que se tiene hacia la región de Puno, el modelo aplicado fue de máxima verosimilitud con la ejecución del modelo Log-Log. Se pudo determinar que las donaciones y transferencias si tienen un impacto positivo hacia la inversión pública de la región de Puno, toda vez que a un incremento de las donaciones y transferencias en $1 \%$ entonces la inversión pública incrementara en $54.48 \%$.

Palabras clave: inversión pública; donaciones y transferencias; salud; transporte. 


\title{
Impact of donations and transfer son public investment in the Puno region, period 2009-2019
}

\begin{abstract}
The objective of the research was to determine the impact of donations and transfers on public investment in the Puno region 2009-2019, given that in recent years, public investment has been questioned mainly because it has not shown significant improvements in the development of the Puno region. A descriptive correlational methodology was applied, considering the database of time series for the analysis of the impact that is had towards the Puno region, the applied model was of maximum likelihood with the execution of the Log-Log model. It was determined that donations and transfers do have a positive impact on public investment in the Puno region, since with an increase in donations and transfers by $1 \%$, then public investment will increase by $54.48 \%$.
\end{abstract}

Keywords: public investment; donations and transfers; health, transportation.

Artículo recibido: 10 Agosto. 2021 Aceptado para publicación: 07. Setiembre. 2021

Correspondencia: jcquispe@unap.edu.pe Conflictos de Interés: Ninguna que declarar 


\section{INTRODUCCIÓN}

Luego de observar los datos estadísticos para el periodo de 2000-2013, se muestra que hubo un importante crecimiento del Producto Interno Bruto (PBI), aumentando alrededor del $50 \%$, esto debido especialmente al incremento de inversiones en proyectos de inversión pública como el sector infraestructura y Social, que se superó de 203 a 1.502 millones de dólares y de 285 a 1.084 millones de dólares. Las inversiones primordiales para el año 2013 fueron los subsectores Hidrocarburos logrando la cantidad de 578 millones de dólares, Transportes fue de a 1.082 millones de dólares, Educación 326 millones de dólares y en Vivienda 404 millones de dólares; afirmando que estos sectores son los que más influyen en el PBI (Cuba \& Yucra, 2020; Quiroz, 2021).

Por lo cual, se determina que en caso de la convergencia absoluta y condicional, no se evidencia una relación directa de las transferencias dentro del proceso de convergencia departamental, para ello es importante que el gobierno destine un mayor presupuesto a la inversión pública a programas orientados a cerrar brechas y para evitar la polarización en la distribución de los ingresos per cápita departamentales, pues en si la realidad es que unos departamentos prosperan y ganan participación en la economía nacional, mientras otros que se mantienen rezagados (Gutierrez, 2015; Perea, 2019; Vázquez-Barquero, 2009)

En diferentes investigaciones se observó que las regiones donde el gobierno nacional tiene el menor apoyo, en general, reciben la mayor parte de los beneficios. Una posible explicación para este resultado se encuentra en el razonamiento de Dixit y Londregan (1996), donde se asume que el gobierno nacional puede tener una ventaja comparativa al dirigir las transferencias fiscales a los grupos de la oposición, transfiriendo a la vez el costo de esta maniobra política a los grupos que lo apoyan (Neyra, 2020).

Existe una gran desigualdad horizontal al momento de hacer transferencias a los gobiernos locales, lo cual al realizar un análisis se tiene la certeza de que la distribución de transferencias tiene varia desigualdad desde las variables sociales; las mismas que se vieron desde diferentes puntos, la clasificación de ingresos y características de los gobiernos regionales, confirman que hay inequidad de distribución de recursos determinados en cuanto a sus transferencias (Valeriano, 2012; Ramírez, 2012; Tecco, 1997) 
Por lo cual se puede establecer que la ejecución de presupuestos en proyectos de inversión, en el departamento de Puno, en los tres niveles de gobierno no han mostrado relevancia para las mejoras de la calidad de vida, en vista que expresada en el Índice de Desarrollo Humano y el porcentaje de pobreza monetaria, estas han mejorado de manera importante en los últimos años pero debido a otros factores como la práctica de las actividades informales y el contrabando (Ministerio de Economía y finanzas, 2010; Orco, 2009; Perea, 2019).

Al considerar este aspecto pero con la distinción de grado de ruralidad de la población, esta es más notoria, ya que los proyectos de inversión pública tienden a cambiar, lo que ocasiona un retraso a los agentes interventores en tanto a la elaboración de proyectos, en los cuales existe intervención participativa en el cual se toma en cuenta la lejanía de la cual proviene, para la elaboración de dichos proyectos deben están en las etapas de identificación y formulación priorizando los que se encuentren en la escala pública (Gonzales \& Franbalt, 2015).

El efecto de las transferencias es diferente por grupos de municipios, toda vez que a mayor incremento de las transferencias, entonces mayor significancia en el gasto público se evidenciará; además, las transferencias por recursos naturales son vistas por los gobernantes locales solo como una transferencia de dinero, sin importar la procedencia y el costo de oportunidad que este representa, el gasto público municipal es representado como una ilusión fiscal, porque los resultados de la investigación de las transferencias gubernamentales mostraron que los municipios en las regiones, en donde las transferencias por recursos naturales per-cápita resultaron negativamente, ya que realizaron un mayor gasto en salud y educación, en la cual concluye que no se requiere de cantidades significativas de las rentas por recursos naturales para ejecutar un gasto público también es importante el capital humano (Canavire-Bacarreza \& Espinoza, 2015; Castro, 2020; Clavellina \& Domínguez, 2020; Nickson, 2002).

Por tanto, el problema identificado es que las transferencias y donaciones no han mostrado un impacto positivo sobre la inversión pública medido a través de ejecución de la inversión pública en sus sectores de gasto. La región de Puno como las otras regiones no reciben una buena distribución de donaciones y transferencias por parte del gobierno central, las donaciones y transferencias que recibe la región de Puno son de fondos financieros no reembolsables, provenientes de Agencias Internacionales, 
gobiernos, instituciones y organismos institucionales, así como de personas naturales y jurídicas. Por ejemplo, en el 2016 se percibieron donaciones y transferencias de S/. 56, 465,163 soles y en el 2019 estas alcanzaron S/. 59, 816,546 soles (MEF, 2020). Las principales fuentes de financiamiento de los proyectos de inversión son un $40 \%$ por recursos determinados, y $42 \%$ de donaciones y transferencias los cuales son administrados por los gobiernos regionales o locales; además, desde el año 2004 existe una tendencia favorable en el presupuesto de inversiones, sin embargo, su distribución no se realiza en base a criterios de carencias o necesidades insatisfechas. Las inversiones en los sectores básicos pudieron cubrir brechas para reducir la pobreza al interior de la región de Puno (MEF, 2020; PACIFICO, 2020; Perea, 2019).

Dada la importancia de la actividad económica manejada en la región Puno y la magnitud referida a las donaciones y transferencias percibidas por la región, es por ello que la presente investigación busco responder las siguientes interrogantes: ¿Cuál es el impacto de las donaciones y transferencias en la inversión pública de la región Puno 2009-2019? ¿Cómo fue el comportamiento de la inversión pública en el sector salud en la región Puno 2009-2019? y ¿Cuál es el efecto de las donaciones y transferencias en los sectores de inversión pública de salud y transportes de la región Puno 2009-2019?.

El objetivo de la presente investigación fue determinar el impacto de las donaciones y transferencias en la inversión pública de la región Puno 2009-2019, además de evaluar el comportamiento de la inversión pública en el sector salud de la región Puno 20092019 y determinar el efecto de las donaciones y transferencias en los en los sectores de inversión pública de salud y transportes de la región Puno 2009-2019.

\section{ESTRATEGIAS METODOLÓGICAS O MATERIALES Y MÉTODOS}

\section{Diseño metodológico}

El presente trabajo se ha definido de tipo no experimental, porque en este contexto, las variables se analizaron desde su estado natural, ya que no existe manipulación para poder observar, contrastar y verificar su comportamiento (Hernández et al., 2014). Para la comprobación de las hipótesis y el cumplimiento de los objetivos planteados en el presente trabajo de investigación, el diseño se basó en definir el impacto de las donaciones y transferencias en la inversión pública de la región Puno 2009-2019. 


\section{Metodología}

El nivel de la investigación es descriptiva, en vista que estas permiten detallar situaciones y eventos, es decir cómo es y cómo se manifiesta determinado fenómeno y busca especificar propiedades importantes de personas, grupos, comunidades o cualquier otro fenómeno que sea sometido a análisis (Hernández, Fernández \& Baptista, 2010). Se define el alcance aun nivel correlacional, debido a que los estudios correlacionales pretender ver como se relacionan o vinculan diversos fenómenos entre sí (o si no se relacionan).

Para evaluar el comportamiento del impacto de las donaciones y transferencias en la inversión pública de la región Puno 2009-2019 se planteó el modelo de Máxima Verosimilitud (MVS), utilizando así el modelo Log-Log, los cuales llegarían a ser logaritmos neperianos con lo cual estos métodos buscan analizar los efectos que tienen las variables independientes sobre la variable dependiente. Este tipo de modelos son como cualquier otro modelo de regresión lineal en el sentido de que los parámetros son lineales. La única diferencia es una de las variables es logarítmica, es un método para analizar los tipos de relación que se pueden establecer entre factores de una tabla de contingencia.

\section{Técnicas}

\section{Revisión documental}

Trabajo que ha permitido una revisión y acopio de información valorativa de documentos y estudios existentes del tema de la investigación, a través de análisis de la información seleccionada lo que constituye un factor relevante para los resultados del trabajo.

\section{Análisis estadístico}

Acción que ha permitido determinar el nivel del impacto de las donaciones y transferencias, realizada en el periodo determinado en la inversión pública, así como su respectivo análisis de los datos estadísticos.

Trabajo que ha permitido una revisión y acopio de información valorativa de documentos y estudios existentes del tema de la investigación, a través de análisis de la información seleccionada lo que constituye un factor relevante para los resultados del trabajo 


\section{Análisis de variables}

\section{Variable Independiente.}

Donaciones y Transferencias: Las transferencias y donaciones provienen de fondos financieros que no son reembolsables recibidos por el gobierno que provienen de Agencias Internacionales de Desarrollo, Gobiernos, Instituciones y Organismos Internacionales, así como de otras personas naturales o jurídicas domiciliadas o no en el país.

Las transferencias están directamente relacionadas con el grado de descentralización, las transferencias tienen que ser hechas a la medida de cada caso, dependiendo de los objetivos deseados, las condiciones iniciales y las limitaciones de recursos. Clasificación de las transferencias fiscales:

Transferencias condicionadas o específicas: El gobierno donante especifica el uso que debe darse a dichos recursos transferidos; en otras palabras, se concede al gobierno beneficiario bajo condición o con la obligación de ser usada solamente para un propósito específico, ya sea que se trate de gastos corrientes, gastos de capital o ambos, es decir, donde su uso esté "amarrado" al propósito o las condiciones del financiamiento. A su vez, las transferencias condicionadas pueden ser obligatorias o discrecionales).

Transferencias no condicionadas: Aquellas que pueden ser gastadas por los gobiernos beneficiarios como si de sus propios recursos tributarios se tratase, es decir, donde el gobierno donante no impone ninguna restricción de uso y el gobierno beneficiario puede decidir libremente sobre el destino de los fondos.

\section{Variable dependiente.}

Inversión Pública: Es la capacidad del estado de aumentar la capacidad económica del País, en la prestación de servicios, mediante la asignación de recursos disponibles en proyectos de inversión pública en el presente para generar un mayor bienestar en el futuro.

La inversión pública se realiza mediante procedimientos técnicos en todas las entidades del Gobierno Nacional, Regional y en el 50\% de los Gobiernos Locales, estas entidades direccionan sus recursos en base a principios, procesos, metodologías y normas técnicas relacionados con el sistema nacional de inversión pública (MEF, Ministerio de Economia y Finanzas, 2020). 
Donde el Sistema Nacional de Inversión Pública, estuvo orientado a mejorar la asignación de los recursos públicos de inversión para el desarrollo, la misma que concentraba la aprobación de proyectos de inversión pública en el Ministerio de Economía y Finanzas (MEF) y a partir del año 2007 en el marco del proceso de descentralización del país, se autoriza la viabilidad de los Proyectos de Inversión Pública a las Oficinas de Programación e Inversiones en cada entidad de gobierno nacional, regional y local, según su competencia y sin límite de monto, para así generar mayores tasas de crecimiento en la inversión pública (Tabla 1).

Tabla 1. Matriz de Variable

\begin{tabular}{|c|c|c|c|c|}
\hline Variables & Factor & $\begin{array}{l}\text { Tipo de } \\
\text { dato }\end{array}$ & $\begin{array}{l}\text { Escala de } \\
\text { medición }\end{array}$ & $\begin{array}{c}\text { Fuente de } \\
\text { información }\end{array}$ \\
\hline $\begin{array}{l}\text { Inversión } \\
\text { publica }\end{array}$ & Económico & Cuantitativo & Millones de soles & \\
\hline $\begin{array}{l}\text { Donaciones y } \\
\text { Transferencias }\end{array}$ & Económico & Cuantitativo & Millones de soles & $\begin{array}{l}\text { MEF - } \\
\text { PERÚ }\end{array}$ \\
\hline $\begin{array}{l}\text { Sector } \\
\text { Transportes }\end{array}$ & Económico & Cuantitativo & Millones de soles & \\
\hline Sector Salud & Económico & Cuantitativo & Millones de soles & \\
\hline
\end{tabular}

\section{RESULTADOS Y DISCUSIÓN}

Análisis del comportamiento de la inversión pública en el sector salud en la región Puno 2009-2019

El comportamiento de la inversión pública en el caso de la región Puno en el año 2009 fue de 800,000,000 soles, ya que en el transcurso de los años la inversión pública ha mostrado un crecimiento sostenible y en los años 2013 hasta el 2016 tuvo un crecimiento regular y en los años 2017 hasta el 2019 la inversión pública mejoro significativamente debido a que han concentrado una mayor participación del presupuesto para la inversión, toda vez que en el año 2019 alcanzo a 1,800,000,000 soles, en el cual se realizó mayor inversión pública. Como se observa en la figura siguiente, las donaciones y transferencias tuvo un impacto significativo en la inversión pública teniendo así un incremento entre los años 2016 al 2018; por tanto, se puede observar que la inversión pública ha tenido una estabilidad considerable (Figura 1). 


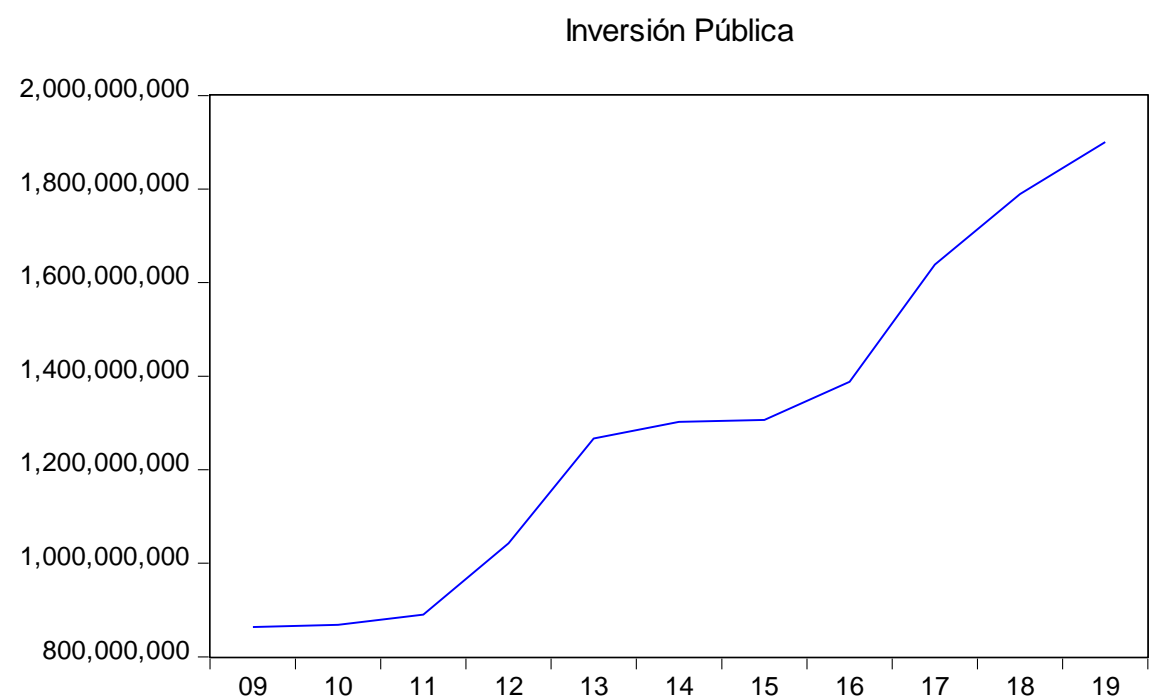

Figura 1. Comportamiento de la inversión pública, 2009 - 2019

El comportamiento de las donaciones y transferencias en la inversión del sector salud para los años 2009 - 2019 en el caso de la Región Puno ha tenido una tendencia variable, llegando a su pico más alto en los años 2018 y 2019, sus picos más bajos en el año 2016 respectivamente por la baja ejecución del presupuesto asignado a inversión de infraestructura y equipamiento, posteriormente hubo un incremento en el año 2018; por lo tanto, se puede observar que en el sector salud no ha tenido estabilidad. En los periodos 2012 a 2015 y 2017 a 2019 tuvieron un crecimiento significativo, regularizando la inversión en los años posteriores hasta el 2019 (Figura 2).

Salud

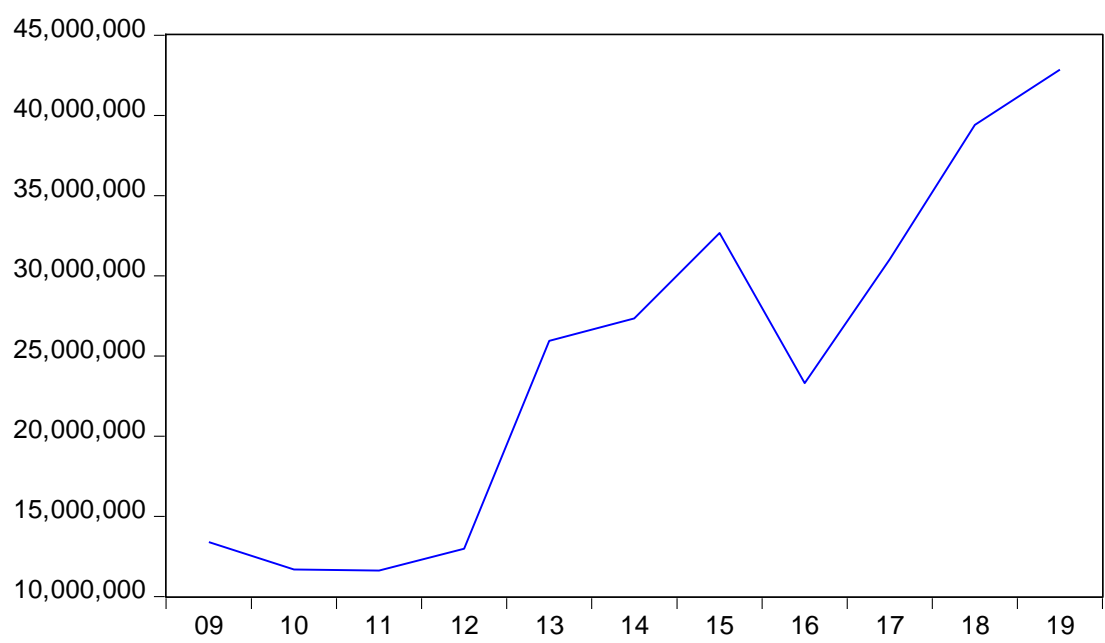

Figura 2. Comportamiento del sector salud, 2009 - 2019 
En el caso del comportamiento que tuvo las donaciones y transferencias en la inversión del sector transporte de la Región Puno, donde fue positiva y creciente entre los periodos 2009 al 2019. La misma que muestra un leve incremento en los años 2016 al 2018 (Figura 3).

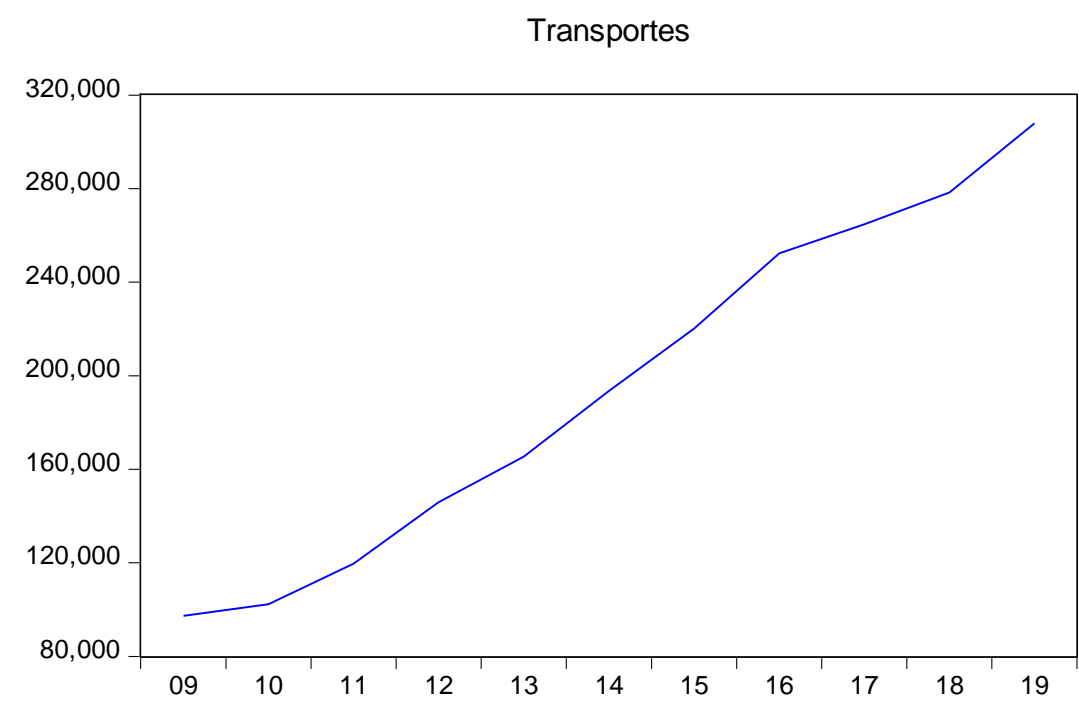

Figura 3. Comportamiento del sector salud, 2009 - 2019

Determinación del impacto de las donaciones y transferencias en la inversión pública de la región Puno 2009-2019

Al plantear la hipótesis nula (Ho), donde las donaciones y transferencias tienen un impacto negativo hacia la inversión pública de la región puno 2009-2019 y la hipótesis alterna (Ha) que las donaciones y transferencias tienen un impacto positivo hacia la inversión pública de la región puno 2009-2019, se consideró el modelo econométrico de tipo Log-Log, obteniendo los siguientes resultados:

Tabla 2. Regresión del modelo de donaciones y transferencias en la inversión pública Variable dependiente: LInversión Pública

\begin{tabular}{lrccc}
\hline \multicolumn{1}{c}{ Variable } & Coefficient & Std. Error & t-Statistic & Prob. \\
\hline Constante & 11.69602 & 1.296368 & 9.022139 & 0.0000 \\
LDonaciones y transferencias & 0.544817 & 0.076327 & 7.137943 & 0.0001 \\
\hline R-squared & 0.849875 & Mean dependent var & 20.94604 \\
Adjusted R-squared & 0.833195 & S.D. dependent var & 0.284546 \\
S.E. of regression & 0.116214 & Akaike info criterion & -1.303805 \\
Sum squared resid & 0.121551 & Schwarz criterion & -1.231460 \\
Log likelihood & 9.170925 & Hannan-Quinn criter. & -1.349408 \\
F-statistic & 50.95024 & Durbin-Watson stat & 1.363534 \\
Prob(F-statistic) & 0.000054 & & \\
\hline
\end{tabular}


De acuerdo a la tabla 2, se puede considerar que las donaciones y transferencias tienen el t-estadístico asciende a 7.13 y es > a 2 (Prob de $0.000<0.05$ ); además, el R cuadrado ajustado es de 83.31, lo que evidencia que las donaciones y transferencias explican a la inversión pública en $83.13 \%$; en este sentido se determina la aceptación de la hipótesis alterna (Ha) y se rechaza la hipótesis nula, por lo que se concluye que las donaciones y transferencias si tienen un impacto positivo hacia la inversión pública de la región puno 2009-2019; del cual a un incremento de las donaciones y transferencias en $1 \%$ entonces la inversión pública incrementara en $54.48 \%$.

\section{Determinación del efecto de las donaciones y transferencias en los sectores de inversión pública salud y transportes de la región Puno 2009-2019}

Para la determinación de los efectos de las donaciones y las transferencias en los sectores de inversión tanto de salud y transportes se procedió la aplicación de una regresión econométrica de tipo Log-Log, del cual los resultados obtenidos fueron:

Tabla 3. Regresión del modelo de donaciones y transferencias en sector salud y transportes

\begin{tabular}{lcc}
\hline \multicolumn{1}{c}{ Variable } & Lsalud & Ltransporte \\
\hline Constante & -0.421820 & -0.834682 \\
\hline Ldonaciones y transferencias & 1.021326 & 0.762349 \\
\hline T-estadístico & 20.43291 & 5.860078 \\
\hline Prob & 0.0000 & 0.0002 \\
\hline R cuadrado ajustado & 0.976554 & 0.769269 \\
\hline
\end{tabular}

En el caso del efecto de las donaciones y las transferencias en el sector salud se puede ver claramente que tiene un efecto positivo, toda vez que este coeficiente es significativo (t-estadístico $=20.43$ o prob $<0.05$ ); por lo cual, a un incremento de $1 \%$ en las transferencias de las donaciones y las transferencias en la región de Puno, entonces la inversión en el sector salud incrementara en 102.13\%. De igual manera el efecto de las donaciones y las transferencias en el sector de transportes tiene un efecto positivo, en vista que su coeficiente es significativo ( $\mathrm{t}$-estadístico $=5.86$ o prob $<0.05$ ); en este sentido, a un incremento de $1 \%$ en las transferencias de las donaciones y las transferencias en la región de Puno, entonces la inversión en el sector transportes incrementara en $76.23 \%$ (Tabla 3 ).

Complementariamente, al realizar la discusión con otros autores, se afirma que el crecimiento económico y la inversión pública, no depende tanto de la eficiencia de 
generar ahorro, cada gobierno realiza movimientos en los cuales generan riqueza para ejecutar mayor gasto público en diferentes sectores a nivel nacional, regional, local y así busque establecer construcciones de infraestructura para el bienestar social de la población, teniendo así un crecimiento mayor al común sino de que el gobierno tendría que establecer condiciones favorables para que la inversión productiva genere riqueza (Hernández, 2010). Además, se define que los ingresos percibidos por las donaciones y transferencias hacia la región impactan de manera positiva a la inversión pública ya que suceden incrementos en la misma los cuales ocasionan crecimiento en los sectores que más impactan a la región como salud y transporte los cuales son de beneficio para la población de la región de Puno con la ejecución de proyectos por parte del sector transporte y seguimientos del cuidado de enfermedades por parte del sector salud todo esto para elevar la calidad de vida de la población en general.

Al igual que en los resultados obtenidos en la presente investigación estas consideran relevancia y también tiene coherencia por lo determinado por Chafloque (2017), donde la ejecución del presupuesto de donaciones y transferencias en el Hospital Regional de Lambayeque del Perú para el factor político dentro de cada institución pública se ejercer a través de un buen desempeño por parte del personal para el buen uso del presupuesto una de estas es la sugerencia de capacitaciones al personal para que así puedan realizar una mejor formulación, ejecución y control de presupuesto para los proyectos a ejecutar Por lo cual, de todas las investigaciones antes mencionadas nos dan a conocer que el que comportamiento que tiene la inversión pública en tanto al sector salud es positivo debido a que se realizan gastos constantes todos años en los cuales se dan los programas de salud para la población como el programa articulado nutricional y la salud materna neonatal y esto coincido con lo determinado por la presente investigación. Con esto la región garantiza el cuidado de las provincias que conforman la misma, para que la población tenga una mejor calidad de vida y cuidados por parte del sector salud de la inversión pública realizada en dicho sector.

En este sentido, los resultados obtenidos en la presente investigación van referidos a que el presupuesto recibido por fuente de donaciones y transferencias hacia la inversión pública para los sectores de salud y transporte son positivos y se desarrollan para un bienestar común de la población y la satisfacción que se tiene por el mejoramiento en ambos sectores, tanto como en infraestructura o adquisición de recursos o bienes. 
Además, determina que las donaciones y transferencia las cuales son ejecutadas con un contribuyente a la nación, estado, municipios e institutos autónomos, estos recursos recibidos son distribuidos según corresponda para diferentes sectores para cada donación o transferencia existe la ley de impuesto sobre sucesiones, Donaciones $\mathrm{N}^{\circ}$ 360, las donaciones o liberalidades podrán ser efectuadas mediante efectivo como también en bienes, ya sean muebles o inmuebles. Contando con la información sobre la liberalidad y donación como el procedimiento que se debe seguir para que sea deducible del impuesto sobre la renta, el cual va a beneficiar no solo a los contribuyentes si no a la colectividad en general (Carhuamaca, 2009; Valenzuela-Reynaga \& Hinojosa-Cruz, 2017).

Finalmente, las inversiones realizadas por parte de la región de puno hacia el sector de transportes son realizadas para el beneficio de la población por medio de mejoramientos de carreteras y construcciones, para que se tenga mejores vías de transporte hacia la región de puno y a todas las provincias que conforman la misma, se puede afirmar que estos gastos en dicho sector son constantes al pasar de los años, debido a que el mejoramiento de las mismas se debe a que son utilizadas constantemente por los vehículos que tienen como fin la región de puno o cualquiera de sus provincias estos sirven como para el movimiento y productividad de las mercancías por parte del comercio internacional de las empresas, el tránsito y el transporte de pasajeros, así como de carga y mercancía en el territorio regional.

\section{CONCLUSIÓN O CONSIDERACIONES FINALES}

De determina que las donaciones y transferencias si tienen un impacto positivo hacia la inversión pública de la región puno 2009-2019, toda vez que a un incremento de las donaciones y transferencias en $1 \%$ entonces la inversión pública incrementara en $54.48 \%$.

Además, en el caso del comportamiento de la inversión pública, en el año 2009 fue de $800,000,000$ soles, ya que en el transcurso de los años la inversión pública ha mostrado un crecimiento sostenible y en los años 2013 hasta el 2016 tuvo un crecimiento regular y en los años 2017 hasta el 2019 la inversión pública mejoro significativamente debido a que han concentrado una mayor participación del presupuesto para la inversión, toda vez que en el año 2019 alcanzo a 1,800,000,000 soles, en el cual se realizó mayor inversión pública. 
Finalmente, el efecto de las donaciones y las transferencias en el sector salud es positivo, toda vez que a un incremento de $1 \%$ en las transferencias de las donaciones y las transferencias, entonces la inversión en el sector salud incrementara en 102.13\%. De igual manera el efecto de las donaciones y las transferencias en el sector de transportes tiene un efecto positivo, ya que a un incremento de $1 \%$ en las transferencias de las donaciones y las transferencias en la región de Puno, entonces la inversión en el sector transportes incrementara en $76.23 \%$.

\section{LISTA DE REFERENCIAS}

Canavire-Bacarreza, G., \& Espinoza, N. G. Z. (2015). Transferencias e impuesto predial en México. Economía UNAM, 12(35), 69-99. https://doi.org/10.1016/j.eunam.2015.09.004

Carhuamaca Mendoza, R. R. (2009). Factores determinantes de la recaudación del impuesto predial en la Municipalidad Provincial de Chupaca, periodo 20092018. In Universidad Nacional del Centro del Perú. Universidad Nacional del Centro del Perú. http://repositorio.uncp.edu.pe/handle/20.500.12894/6083

Castro, E. B. G. (2020). Incidencia de la asignación de transferencias en la recaudación fiscal en los municipios del Perú. Revistas.Unap.Edu.Pe, 9(2), 1503-1517. https://doi.org/10.26788/riepg.2020.2.167

Chafloque Farfán, C. (2017). El presupuesto por resultados y sus efectos en la calidad del gasto del sector salud de la Región Lambayeque 2011-2016. Universidad César Vallejo.

Clavellina Miller, J. L., \& Domínguez Rivas, M. I. (2020). Implicaciones económicas de la pandemia por COVID-19 y opciones de política. http://www.ibd.senado.gob.mx/

Cuba Espinoza, F., \& Yucra Vargas, A. R. (2020). Las tendencias demográficas y su relación con la pobreza en el Perú, 2000 - 2017. Universidad Andina Del Cusco. http://repositorio.uandina.edu.pe:8080/xmlui/handle/UAC/3589

Fernando Valeriano Ortíz, L. (2012). La modernización de la gestión pública en el Perú. In revistasinvestigacion.unmsm.edu.pe

(Vol.

$15)$. https://revistasinvestigacion.unmsm.edu.pe/index.php/administrativas/article/vie $\mathrm{w} / 8802$

Gutierrez Sandoval, L. L. (2015). Gestion Del Presupuesto Por Resultados Y Su 
Influencia En La Calidad De Gasto Publico En La Red Salud Sanchez Carrion 2014.

Hernández, Fernández, \& Baptista. (2014). Metodología de la investigación (McGraw Hill.

https://dspace.scz.ucb.edu.bo/dspace/bitstream/123456789/166/1/1646.pdf

Hernández Mota, J. L. (2010). Inversión pública y crecimiento económico: Hacia una nueva perspectiva de la función del gobierno. In scielo.org.mx. http://www.scielo.org.mx/scielo.php?pid=S0188-

$33802010000200003 \&$ script $=$ sci_abstract $\&$ tlng $=$ en

Hernández Sampieri, R., Fernández Collado, C. \& Baptista Lucio, P. (2010). Metodología de la investigación. http://www.academia.edu/download/38911499/Sampieri.pdf

$\begin{array}{llll}\text { MEF. } & \text { (2020). } & \text { Memorial }\end{array}$ https://www.smv.gob.pe/ConsultasP8/temp/Memoria 2019 - 1.pdf

Ministerio de Economía y finanzas. (2010). Proyecto de Inversión pública. Ministerio de Economía $\quad y \quad$ Finanazas, 307. https://www.mef.gob.pe/contenidos/conta_publ/2010/tomo1/6_inversion_public a.pdf

Neyra López, C. (2020). Caracterización de la región Puno 2020. Sistema Nacional de Evaluación, Acreditación y Certificación de La Calidad Educativa - SINEACE. http://repositorio.sineace.gob.pe/repositorio///handle/sineace/6226

Nickson, A. (2002). Transferencia de políticas y reforma en la gestión del sector público en América Latina: el caso del New Public Management. Revista Del CLAD Reforma $\quad y \quad$ Democracia. http://www.sidalc.net/cgibin/wxis.exe/?IsisScript=COLEC.$x i s \&$ method=post $\&$ formato $=2 \&$ cantidad $=1 \&$ e $\mathrm{xpresion}=\mathrm{mfn}=004436$

Orco Díaz, A. (2009). Gasto público en inversiones y reducción de la pobreza regional en el Perú, periodo 2009-2018. 28, 2009-2018. https://doi.org/10.15381/quipu.v28i56.17087

PACIFICO, U.;MEF. (2020). BALANCE DE LA INVERSIÓN PÚBLICA: AVANCES Y DESAFÍOS PARA CONSOLIDAR LA COMPETITIVIDAD Y EL BIENESTAR DE LA POBLACIÓN Estudio realizado por la Universidad 
Del Pacífico por encargo de la Dirección de Programación Multianual del Sector $\begin{array}{llll}\text { Público del } & \text { Ministerio. }\end{array}$ https://www.mef.gob.pe/contenidos/inv_publica/docs/estudios_documentos/estu dios/Estudio_Balance_de_la_Inversion_Publica.pdf

Perea Flores, H. (2019). Fuentes de Financiamiento del Presupuesto del Sector Público 2019. Congreso de La Republica. http://www.congreso.gob.pe/Docs/comisiones2018/Presupuesto/files/vice_econo mia_mef.pdf

Quiroz Lozada, A. J. A. (2021). Impacto del crecimiento económico en la pobreza del Perú período 2007 - 2018. Repositorio Institucional - USS. http://repositorio.uss.edu.pe//handle/20.500.12802/7640

Ramírez Viveros, J. (2012). La política pública de participación ciudadana en el gobierno local: caso Alcobendas, España. Scielo.Org.Mx. http://www.scielo.org.mx/scielo.php?pid=S187035692012000100005\&script=sci_arttext\&tlng=en

Tecco, C. (1997). El gobierno municipal como promotor del desarrollo local-regional. In municipios.unq.edu.ar. http://municipios.unq.edu.ar/modules/mislibros/archivos/gobloc_desloc.pdf

Valenzuela-Reynaga, R., \& Hinojosa-Cruz, A. V. (2017). Las transferencias federales, los contrapesos políticos y los ingresos fiscales estatales en México. Economía UNAM, 14(42), $47-71$. https://doi.org/https://doi.org/10.1016/j.eunam.2017.09.002

Vázquez-Barquero, A. (2009). DESARROLLO LOCAL, UNA ESTRATEGIA PARA TIEMPOS DE CRISIS. In CONCEPTOS CRITICOS Universitas Forum (Vol. 1, Issue 2). http://municipios.unq.edu.ar/modules/mislibros/archivos/barquero_UF2.pdf 